\title{
Offener Call for Papers
}

Die Redaktion der Vierteljahreszeitschrift integration lädt Autoren aus den Politik-, Wirtschafts-, Rechts- und Geschichtswissenschaften sowie angrenzenden Disziplinen, die sich mit Grundfragen der europäischen Integration (Stand und Perspektiven der Europäischen Union, Reformbedarf, die Europäische Union nach Lissabon, differenzierte Integration) und der aktuellen Europapolitik (Politikfelder wie Europapolitik der Mitgliedstaaten) wissenschaftlich beschäftigen, dazu ein, Aufsätze zum Gutachterverfahren einzureichen. Insbesondere möchte die Redaktion Nachwuchswissenschaftler ermuntern, aktuelle Ergebnisse aus ihrer Forschung für eine Veröffentlichung anzubieten.

Die integration wird von einem Herausgebergremium unter Vorsitz von Prof. Dr. Heinrich Schneider für das Institut für Europäische Politik e.V. in Zusammenarbeit mit dem Arbeitskreis Europäische Integration e.V. herausgegeben. Sie richtet sich an alle, die sich in Wissenschaft, Bildung, Politik, Administration und in den Medien mit europäischen Fragen beschäftigen. Als ein theoriegeleitetes und politikbezogenes interdisziplinäres Forum bietet sie Raum, um Fragen der europäischen Integration und EU-Politik zu diskutieren.

Eingereicht werden sollen deutschsprachige Aufsätze mit einem Umfang von 40.000 bis 50.000 Zeichen (inklusive Leerzeichen und Anmerkungen), die sich an ein interdisziplinäres Publikum richten. Voraussetzung ist, dass die Manuskripte unveröffentlicht und nicht zugleich an anderer Stelle eingereicht sind. Manuskripte, die bereits in einer grauen Reihe oder im Internet veröffentlicht sind, können nur in einer substanziell überarbeiteten Fassung in das Gutachterverfahren aufgenommen werden. Hinweise zur Gestaltung des Manuskripts finden Sie im Merkblatt für Autoren, das unter http://www.iep-berlin.de/autorenhinweise.html abrufbar ist.

Über die Veröffentlichung der eingesandten Aufsätze wird nach Abschluss eines Gutachterverfahrens entschieden. Über den Zeitpunkt der Veröffentlichung entscheidet die Redaktion.

Fristen für die Einreichung sind:
Ausgabe 4/2013:
1. Juni 2013
Ausgabe 1/2014:
1. September 2013
Ausgabe 2/2014:
1. Dezember 2013

Einreichungen:

Als Word-Datei bitte an julian.plottka@iep-berlin.de

\section{Rückfragen:}

Richten Sie bitte an Julian Plottka

E-Mail: julian.plottka@iep-berlin.de

Telefon: +49 / 30 / 88913484 